\title{
Very Simple BICM-ID Using Repetition Code and Extended Mapping with Doped Accumulator
}

\author{
Khoirul Anwar • Tad Matsumoto
}

Published online: 13 September 2011

(C) The Author(s) 2011. This article is published with open access at Springerlink.com

\begin{abstract}
This paper proposes very simple bit-interleaved coded modulation with iterative detection (BICM-ID) as a spectrally efficient signal transmission scheme, where irregular repetition code and extended mapping with rate-1 doped accumulator (ACC) are combined to achieve a clear turbo-cliff. Doped ACC is used for close matching of the demapper and decoder's extrinsic information transfer curves. Although the proposed BICM-ID system is very simple, it can achieve excellent performances and completely eliminate bit-errorrate floor. An exemplifying result for extended non-Gray mapped 4-quadrature amplitude modulation symbol shows that with 0.916 bits/channel use, turbo-cliff happens at $1.27 \mathrm{~dB}$ away from the Shannon limit.
\end{abstract}

Keywords BICM-ID $\cdot$ Extended mapping $\cdot$ Iterative decoding $\cdot$ Repetition code

\section{Introduction}

Bit-interleaved coded modulation with iterative detection/decoding (BICM-ID) [1,2] has been recognized as being a bandwidth efficient signal transmission scheme, where in the transmitter, the encoder and the mapper are separated by an interleaver. Iterative detectionand-decoding takes place at the receiver by exchanging the extrinsic log-likelihood ratio

This research is supported in part by the Japan Society for the Promotion of Science (JSPS) Grant under Scientific Research (C) No. 22560367 and (B) No. 23360170.

K. Anwar $(\varangle) \cdot$ T. Matsumoto

School of Information Science, Japan Advanced Institute of Science and Technology (JAIST),

1-1 Asahidai, Nomi, Ishikawa 923-1292, Japan

e-mail: anwar-k@jaist.ac.jp

T. Matsumoto

Center for Wireless Communication, University of Oulu, 90014 Oulu, Finland

e-mail: tadashi.matsumoto@ee.oulu.fi; matumoto@jaist.ac.jp 
(LLR), obtained as a result of maximum a posteriori probability (MAP) algorithm for demapping/decoding via interleaver/deinterleaver, following the standard turbo principle.

In principle, since the BICM-ID is a serially concatenated system, the analysis of its performance can rely on the area property of extrinsic information transfer (EXIT) chart. If the area between the EXIT curves of the demapper and the decoder is made very small while keeping the tunnel open until a point very close to the $(1,1)$ mutual information (MI) point, system can achieve performance closer to the Shannon limit with a clear turbo-cliff. Therefore, the transmission link design based on BICM-ID falls into the issue of matching between the demapper and the decoder EXIT curves.

Various techniques have been proposed for better matching between the demapper and decoder in BICM-ID without requiring heavy detection/decoding complexity aiming at achieving near-Shannon limit performance. One of the techniques that can provide us with design flexibility is extended mapping (EM). EM allocates more than $m$-bits to one signal point in $2^{m}$-quadrature amplitude modulation (QAM) format [1,3]. With EM, the demapper EXIT curve exhibits sharper decay, where the left-most point of the demapper EXIT curve has a lower value than the demapper with Gray mapping, but the right-most point becomes higher. With the sharper decay of the demapper EXIT curve, relatively weak codes such as a memory-1 convolutional code (CC) better matches the EM demapper than strong codes such as Turbo codes [3].

References [1] and [4] propose the use of even weaker code, irregular repetition codes, where the degree distribution for the IRC with different variable node degrees $\left(d_{v_{i}}\right)$ in one coded block are determined empirically to minimize the gap. Quite recently in [5], the authors have shown that determining the optimal degree distribution falls into a simple convex optimization problem, solvable by using a linear programming technique.

Despite the simplicity of the BICM-ID system proposed by $[1,4,5]$, however, it has a fundamental drawback that it still suffers from the error floor. This is because the demapper EXIT curve does not reach a point very close to the $(1,1)$ MI point.

In this paper, we use doped accumulator (ACC) [6] on the top of the BICM-ID structure presented in $[1,4]$, such that the EXIT curves are more closely matched, but do not intersect each other, until a point close enough to the $(1,1)$ MI point such that the EXIT trajectory can sneak-through the convergence tunnel. Another advantage of using doped ACC is that we can achieve a higher spectrum efficiency by eliminating the necessity of the use of single parity check code (SPC), which is necessary in [1] to reduce the error floor.

The system structure proposed in this paper is shown in Fig. 1. It is found that with the doping ratio $(1: P)=(1: 1)$ the structure has a similarity to irregular repeat accumulate (IRA) ${ }^{1}$ coded BICM-ID with the check node degree $d_{c}$ being one, where $\Pi$ is a random interleaver. The smaller the $P$ value the lower the left-most part of the EXIT curve of the combined demapper and ACC, for which it is unavoidable that intersection between demapper and the decoder EXIT curves happens at low MI point. Therefore, the doping ratio $(1: P)$ should be carefully chosen. $d_{c}=1$ is acceptable because information bits are directly connected to the extended mapper, by which LLRs of certain bits at the output of the demapper are not forced to be zero. Furthermore, $d_{c}=1$ does not sacrifice the spectrum efficiency in term of bits-per-channel use because the EM rule, specified by $\mu$, allocates more than $m$ bits to one signal constellation point.

A goal of this paper is not to present a capacity-approaching BICM-ID technique as in [7], but to show that with the proposed BICM-ID having a very simple structure, clear turbo-cliff

1 However, it is not similar to IRA when $P>1$. 
(without error floor) can still happen at a signal-to-noise power ratio (SNR) relatively close to the Shannon limit for for a given code rate.

This paper is organized as follows. Section 2 proposes the structure of EM BICM-ID with doped ACC and also describes the labeling pattern with several mapping schemes. Section 3 analyzes the convergence properties of the proposed scheme using EXIT chart. Section 4 evaluate the bit-error-rate (BER) performances of the proposed structure. Finally, Sect. 5 concludes the paper with some remarks.

\section{Proposed Simple Structure}

\subsection{Transmitter}

As shown in Fig. 1, a binary bit information sequence $b$ to be transmitted is encoded by IRC to obtain the coded sequence $x$, where the repetition times take several different values in one block, represented as $d_{v_{i}}$. By letting $a_{i}$ denote the distribution of the node degree $d_{v_{i}}$, the length of IRC coded block is $K=\sum_{i} a_{i} \cdot d_{v_{i}} \times N$, which is equal to the random interleaver length, yielding the code rate of $\mathcal{R}=N / K$, where $N$ is the information length.

Repetition coded sequence $x$ is bit-interleaved, resulting in time-permutated sequence $y$. The sequence $y$ is doped accumulated with doping ratio of $(1: P)$. The doped ACC is a systematic recursive convolutional code (SRCC) with coding rate $R=1$. To keep the doped ACC's code rate equal to one, the uncoded bit $c_{u}(k)$ at every $k=n P$ th bit is replaced by the doped ACC-coded bit $c_{c}(k)$, which is the output of accumulated $P$ interleaved repetition coded bits. With $n=1,2, \ldots, K / P$ and $k$ being the bit index, the sequence $c$ is expressed as

$$
c(k)= \begin{cases}c_{c}(k) & \text { if } k=n P \\ c_{u}(k) & \text { otherwise. }\end{cases}
$$

With EM, $\ell$ bits are mapped to one of the $2^{m}$ constellation for modulation. Since $\ell>m$ with EM, more than one labels having different bit patterns in the segment are mapped on to each constellation point. However, there are many possible combinations of the labeling patterns, and hence determining the optimal labeling pattern plays a crucial role towards achieving near-Shannon limit performance.

This paper considers the optimized labeling which is extended non-Gray mapping such that with full a priori information, the MI between the IRC-coded transmitted bit and the demapper output LLR is maximized. Figure $2 \mathrm{a}, \mathrm{b}$ show the labeling pattern with $\ell=2$ for normal Gray and non-Gray mapping, respectively. Extended mapping with $\ell=\{3,4,5\}[1]$ are shown in Fig. 2c, d, and e, respectively, where each 4-QAM symbol constellation point has $2^{\ell-m}$ labels.

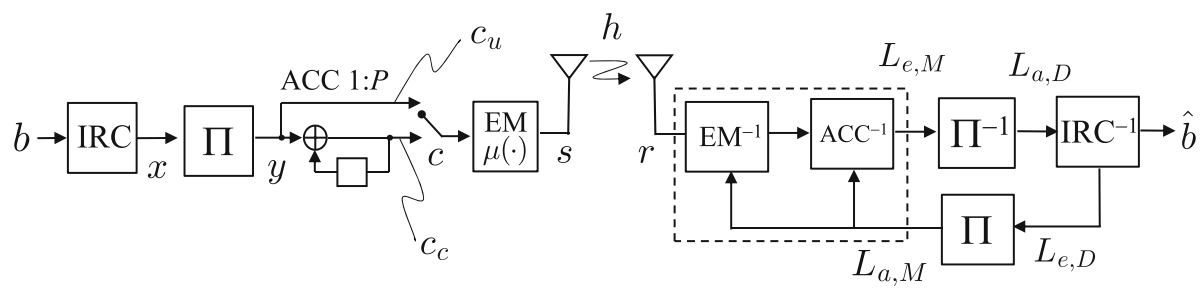

Fig. 1 Structure of EM BICM-ID with doped ACC and IRC 


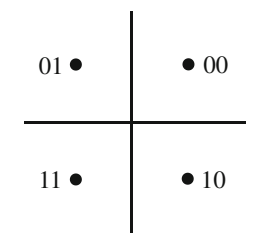

(a) Gray $(\ell=2)$

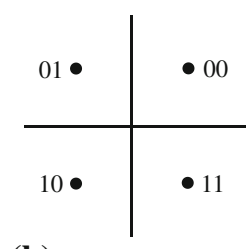

(b) Non-Gray $(\ell=2)$

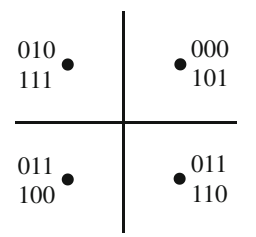

(c) $\mathrm{EM}(\ell=3)$

\begin{tabular}{l|r}
1101 & 0100 \\
1011 & 1110 \\
0111 & $\bullet 0010$ \\
0001 & 1000 \\
\hline 1010 & 1111 \\
$1100 \bullet$ & $\bullet 0101$ \\
0000 & 1001 \\
0110 & 0011
\end{tabular}

(d) $\operatorname{EM}(\ell=4)$

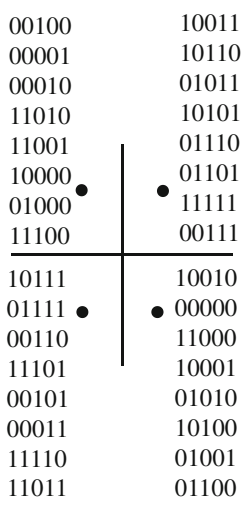

(e) $\operatorname{EM}(\ell=5)$

Fig. 2 Symbol labeling patterns for EM 4-QAM: a Gray $(\ell=2)$, b Non-Gray $(\ell=2)$, c EM $\ell=3$, d $\mathrm{EM} \ell=4$, e $\mathrm{EM} \ell=5$

The ambiguity of bit identification at the receiver due to the $2^{\ell-m}$ labels, allocated into one constellation point, can be avoided by the use of a priori information provided by the decoder, at each iteration, in the form of LLR [8]. The convergence behavior of the iterative detection/decoding techniques can be evaluated using EXIT chart [8]. The impact of labeling pattern appears in the shape of EXIT curve representing relationship of a priori and extrinsic of MI between the coded sequence $c$ and its corresponding LLR, as shown in Subsect. 2.4.

The $\ell$ coded bits in sequence $c$ are grouped to form a label for EM

$$
q=\left[c_{1}, c_{2}, \ldots, c_{v}, \ldots, c_{\ell}\right],
$$

which is mapped to QAM symbol of

$$
s=\mu(q)
$$

according to the mapping rule $\mu(\cdot)$. The EM-mapped symbols $s$ is then transmitted over the channel.

\subsection{Channel}

Additive white Gaussian noise (AWGN) channel having gain $h$ is assumed. The received signal is then expressed as

$$
r=h s+\eta,
$$

where $\left\langle|s|^{2}\right\rangle=1$, and $\eta$ is an independent and identically distributed (i.i.d) complex zeromean Gaussian random variable with variance $\sigma^{2}=10^{-\mathrm{SNR}[\mathrm{dB}] / 10}$.

\subsection{Receiver}

Iterative demapping and decoding process is invoked, where the extrinsic information is exchanged iteratively between the demapper and decoder. Decoding of ACC, referred to as $\mathrm{ACC}^{-1}$, is performed by using Bahl-Cocke-Jelinek-Raviv (BCJR) algorithm after EM demapping $\left(\mathrm{EM}^{-1}\right)$ and before de-interleaving $\Pi^{-1}$. Decoding of irregular repetition code 


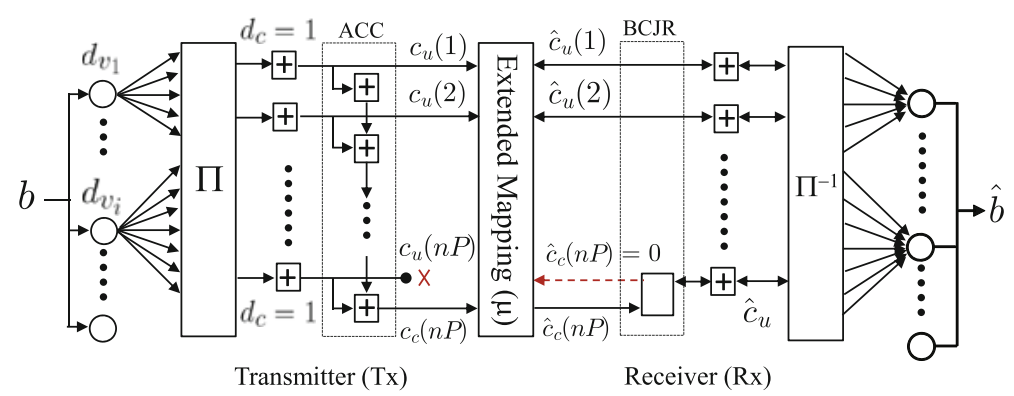

Fig. 3 Tanner graph of BICM-ID using IRC and EM with doped ACC

$\left(\mathrm{IRC}^{-1}\right)$ is then performed and the iteration continues until no more relevant gains in extrinsic LLR can be achieved. ${ }^{2}$ When the convergence point is reached, binary decision is made on the information bits $\hat{b}$, based on the a posteriori LLR at each $d_{v_{i}}$. Obviously, keeping the convergence tunnel open until the $(1,1)$ MI point is necessary to achieve arbitrarily low BER.

With the help of a priori information $L_{a, M}$ provided by the IRC ${ }^{-1}$ decoder, the demapper calculates the extrinsic LLR $L_{e, M}$ of the bit $q[v]$ in (2) from the received signal $r$ by

$$
\begin{aligned}
L_{e, M}(q[v]) & =\ln \frac{P(q[v]=0 \mid r)}{P(q[v]=1 \mid r)} \\
& =\ln \frac{\sum_{s \in s_{0}} \exp \left\{-\frac{|r-h s|^{2}}{\sigma^{2}}\right\} \prod_{w=1, w \neq v}^{\ell} \exp \left\{-q[w] L_{a, M}(q[w])\right\}}{\sum_{s \in s_{1}} \exp \left\{-\frac{|r-h s|^{2}}{\sigma^{2}}\right\} \prod_{w=1, w \neq v}^{\ell} \exp \left\{-q[w] L_{a, M}(q[w])\right\}},
\end{aligned}
$$

where $s_{0}, s_{1}$ indicates the set of labels in (3) having bit $q[v]$ being 0 and 1 , respectively. $L_{a, M}(q[v])$ is the a priori LLR fed back from the decoder $\operatorname{IRC}^{-1}$ corresponding to the $v$ th bit position in the label $q$ allocated to the signal point $s$.

The output extrinsic LLR of the demapper is then updated by the BCJR algorithm. LLR of every $k=n P$ th coded bit, $\hat{c}_{c}(n P)$, is substituted by the LLR of the uncoded bit $\hat{c}_{u}(n P)$ which is obtained from the $\mathrm{ACC}^{-1}$, as shown in a Tanner graph of the proposed system in Fig. 3.

The extrinsic LLR of demapper output, $L_{e, M}$, is de-interleaved to obtain $L_{a, D}$. For the $d_{v_{i}}$ bits connected to one variable node, the extrinsic LLR is updated by summing up the LLR for the $\left(d_{v_{i}}-1\right)$ bits, as

$$
L_{e, D}(j)=\sum_{J=1, j \neq j}^{d_{v_{i}}} L_{a, D}(\jmath),
$$

to produce extrinsic LLR of the $j$ th IRC-coded bit.

However, because IRC $^{-1}$ does not produce LLR corresponding to the ACC-coded output bits $\hat{c}_{c}(n P)$, for the sake of complexity reduction, the feedback $\hat{c}_{c}(n P)$ from the decoder to the demapper is set at zero, as shown by Fig. 3.

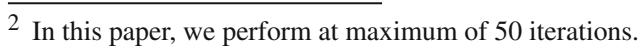




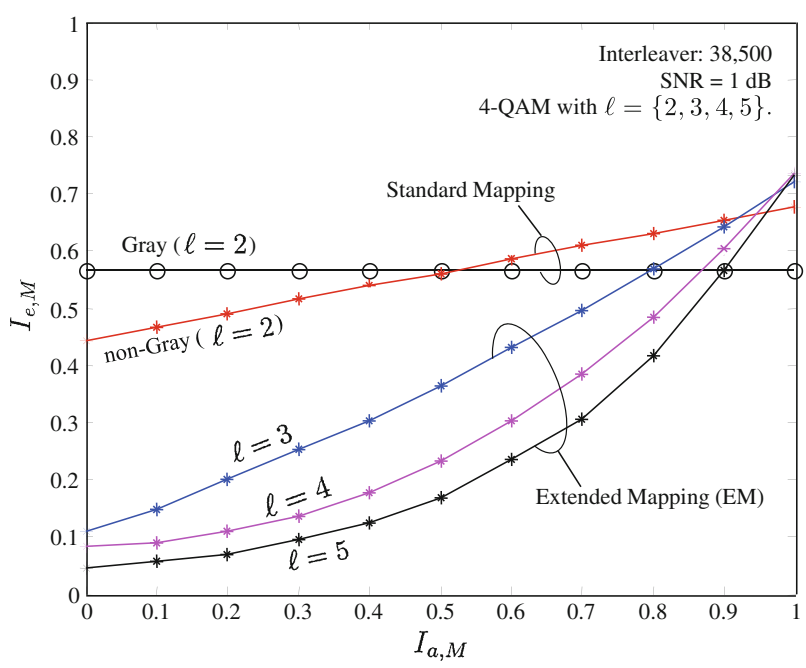

Fig. 4 EXIT curves of EM demapper for several $\ell$

\subsection{Demapper and Mapping Pattern}

Figure 4 compares for SNR $=1 \mathrm{~dB}$ the EXIT functions of 4-QAM demapper for several mapping schemes. The $X$-axis, $I_{a, M}=I\left(c ; L_{a, M}\right)$, is the a priori MI between coded sequence $c$ and $a$ priori LLR $L_{a, M}$ provided by the decoder, while $Y$-axis, $I_{e, M}=I\left(c ; L_{e, M}\right)$, is the MI between $c$ and its corresponding extrinsic LLR $L_{e, M}$ to be forwarded to the decoder. A mapping with $\ell=5$ means that each 4-QAM symbol has 5 bits resulting in 8 labeling patterns allocated to each constellation point.

It can be observed from Fig. 4 that the standard Gray mapping is flat, while standard nonGray mapping (with $\ell=2$ ) has decay. From the EXIT curves shown in Fig. 4, it is found that standard/non-extended mapping best matches turbo codes [9] and/or convolutional codes with large memory, while the extended mapping $(\ell>2)$ is better matched with the repetition codes such as the code proposed in [1]. The matching analysis between the mapper and encoder is further discussed using EXIT analysis provided by Sect. 3.

\section{EXIT Analysis of the Proposed Technique}

With the Gaussianity assumption of the LLRs distribution, let the variance of LLRs $L_{a, M}$ and $L_{e, M}$, a priori and extrinsic LLR of demapper (including $\mathrm{ACC}^{-1}$ ), respectively, be denoted by $\sigma_{L_{a, M}}$ and $\sigma_{L_{e, M}}$. The combined EXIT function of the demapper and $\mathrm{ACC}^{-1}$, denoted as $T_{M}$, is then expressed by

$$
I_{e, M}=T_{M}\left(I_{a, M}, \mathrm{SNR}\right)
$$

where

$$
I_{e, M}=J\left(\sigma_{L_{e, M}}\right) \quad \text { and } \quad I_{a, M}=J\left(\sigma_{L_{a, M}}\right) \text {. }
$$


$J(\cdot)$ and $J^{-1}(\cdot)$ are the functions that convert variance of LLR $\sigma_{L}$ to its corresponding MI, and its inverse, respectively, given by [8]

$$
\begin{aligned}
J\left(\sigma_{L}\right) & =\left(1-2^{-H_{1} \sigma_{L} 2 H_{2}}\right)^{H_{3}}, \\
J^{-1}\left(I_{a}\right) & =\left(-\frac{1}{H_{1}} \log _{2}\left(1-I_{a}^{\frac{1}{H_{3}}}\right)\right)^{\frac{1}{2 H_{2}}},
\end{aligned}
$$

where $H_{1}=0.3073, H_{2}=0.8935, H_{3}=1.1064$, and $\sigma_{L}=\left\{\sigma_{L_{a}, M}, \sigma_{L_{e}, M}\right\}$.

The EXIT function of IRC $^{-1}$, denoted as $T_{D}$, is then given by

$$
\begin{aligned}
I_{e, D} & =T_{D}\left(I_{a, D}\right), \\
& =\frac{\sum_{i} a_{i} \cdot d_{v_{i}} \cdot J\left(\sqrt{\left(d_{v_{i}}-1\right) \cdot J^{-1}\left(I_{a, D}^{i}\right)^{2}}\right)}{\sum_{i} a_{i} \cdot d_{v_{i}}},
\end{aligned}
$$

where $I_{a, D}^{i}$ is the a priori MI of variable node with the $i$ th degree and $I_{e, D}$ is its output extrinsic MI.

Figure 5 shows for $\mathrm{SNR}=\mathcal{R} \cdot E_{b} / N_{0}=1 \mathrm{~dB}$ the EXIT curves of the extended non-Gray 4-QAM demapper and the decoder used in [1,4,5], where 4-QAM mapping with $\ell=5$ is assumed. The node degree distribution is $d v_{1}=5\left(a_{1}=0.77\right), d v_{2}=7\left(a_{2}=0.23\right)$, without SPC.

According to the area property, the Shannon limit is approached by minimizing the gap between the two curves $T_{M}$ and $T_{D}^{-1}$ (defined in Eq. 11), while keeping the convergence tunnel open until a point very close enough to the $(1,1)$ MI point. It is shown in [1] that with the empirically obtained (near optimum) variable node degree distribution, the intersection between $T_{M}$ and $T_{D}^{-1}$ is at $I_{a, M}=I_{e, D}=0.992$. Since at high a priori $\mathrm{MI}$ the a posteriori LLR is almost the same as the extrinsic LLR, $I_{p, D} \approx I_{e, D}=0.992$. Hence, the bit-error-rate (BER) can be predicted as [8]

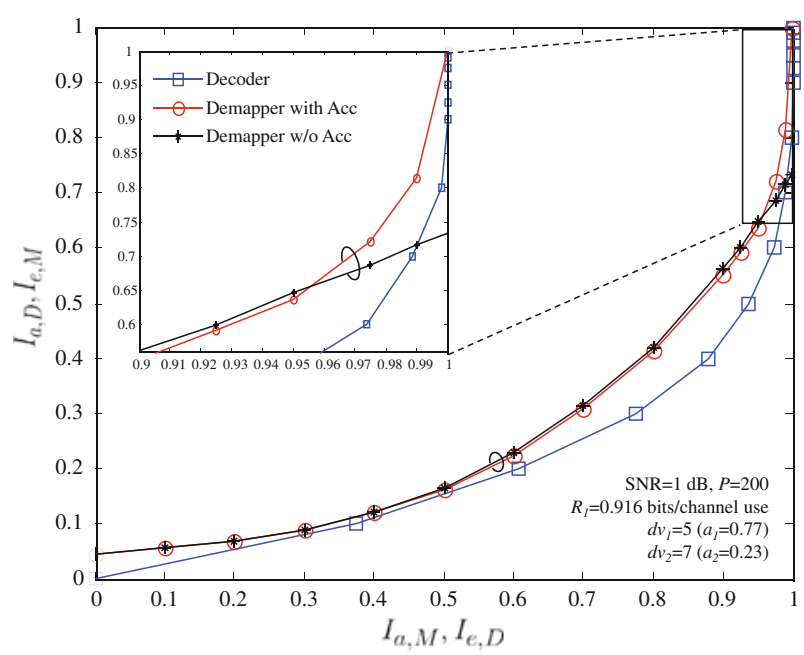

Fig. 5 EXIT charts of IRC without SPC and extended non-Gray 4-QAM with $\mathcal{R}_{1}=0.916$ bits/channel use at $\mathrm{SNR}=1 \mathrm{~dB}$ and doping ratio of $(1: 200)$ 


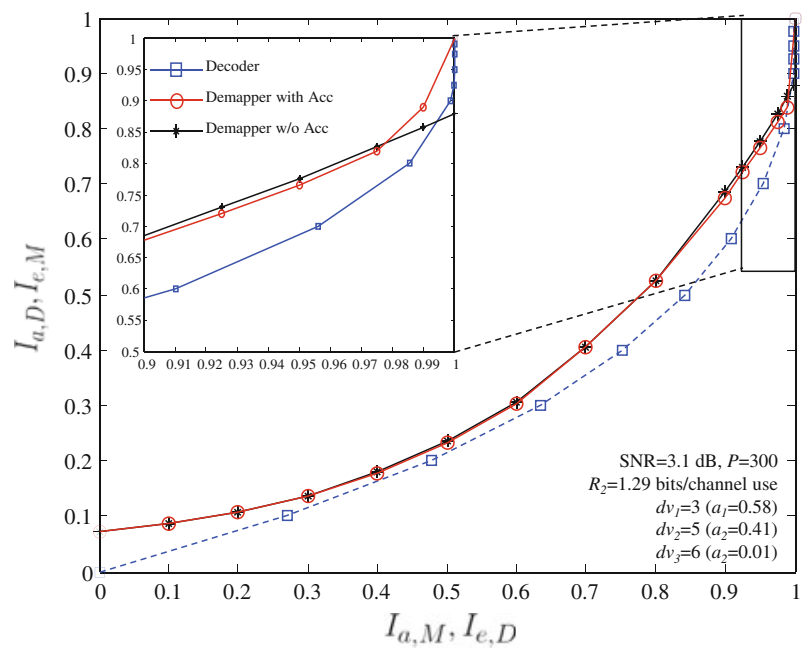

Fig. 6 EXIT charts of IRC without SPC and extended non-Gray 4-QAM with $\mathcal{R}_{2}=1.299$ bits/channel use at $\mathrm{SNR}=3.1 \mathrm{~dB}$ and doping ratio of $(1: 300)$

$$
\begin{aligned}
\operatorname{BER} & =\frac{1}{2} \operatorname{erfc}\left(\frac{J^{-1}\left(I_{p, D}\right)}{2 \sqrt{2}}\right) \\
& \approx 1.9 \times 10^{-3}
\end{aligned}
$$

It is also suggested from (12) that the required MI to achieve BER of $10^{-5}$ is $I_{p, D}=0.99994$. Ref. [1] proposes the use of SPC concatenated with IRC to push the $T_{D}^{-1}$ curve to the right hand side such that the intersection happens at a point close to $(1,1)$ MI point. However, the use of doped ACC with doping ratio of (1:200) bends the shape of the $T_{M}$ curve such that it can reach a point very close to the $(1,1)$ MI point, resulting in better matching between the two EXIT curves. Furthermore, the intersection of the two curves can be avoided, as shown in Fig. 5. Hence, the error floor can be completely removed. In this figure, we used the doping ratio of (1:200). It can be observed that the combined use of IRC and doped ACC makes the demapper and decoder curves better matched than IRC alone for the BICM-ID technique.

The rate of IRC can be very flexibly changed by determining the distribution of $d_{v_{i}}$ to provide a turbo-cliff at a desired SNR. EXIT curves of an EM 4-QAM demapper and decoder, designed for turbo-cliff to happen at around SNR $=3.1 \mathrm{~dB}$ is shown in Fig. 6, of which the degree distribution is shown in the figure. Without doped ACC, the EXIT curves with the demapper and the decoder intersect at around $I_{e, D}=0.995$, where the predicted BER, according to (12), is about $1.136 \times 10^{-3}$. However, the use of doped ACC with doping ratio (1:300) changes the shape of demapper EXIT curve to reach a point very close to $(1,1) \mathrm{MI}$ point, and hence no error floor is expected.

\section{Performances Evaluation}

A series of computer simulation was conducted to evaluate the performance and the convergence property of the proposed technique. To obtain reliable enough results, we transmitted 100 blocks, each having 10,000 information bits. We consider random interleaver/deinterleaver with a length of $K=\sum_{i} a_{i} \cdot d_{v_{i}} \times 10,000$. The doping parameters for ACC is set 
Table 1 Node degree distribution for Case 1 and Case 2

\section{Case 1}

$d_{v_{1}}=5\left(a_{1}=0.77\right)$

$d_{v_{2}}=7\left(a_{2}=0.23\right)$

Interleaver length $K=54,600$

$d_{v_{1}}=3\left(a_{1}=0.58\right)$

$d_{v_{2}}=5\left(a_{2}=0.41\right)$

$d_{v_{3}}=6\left(a_{3}=0.01\right)$

Interleaver length $K=38,500$

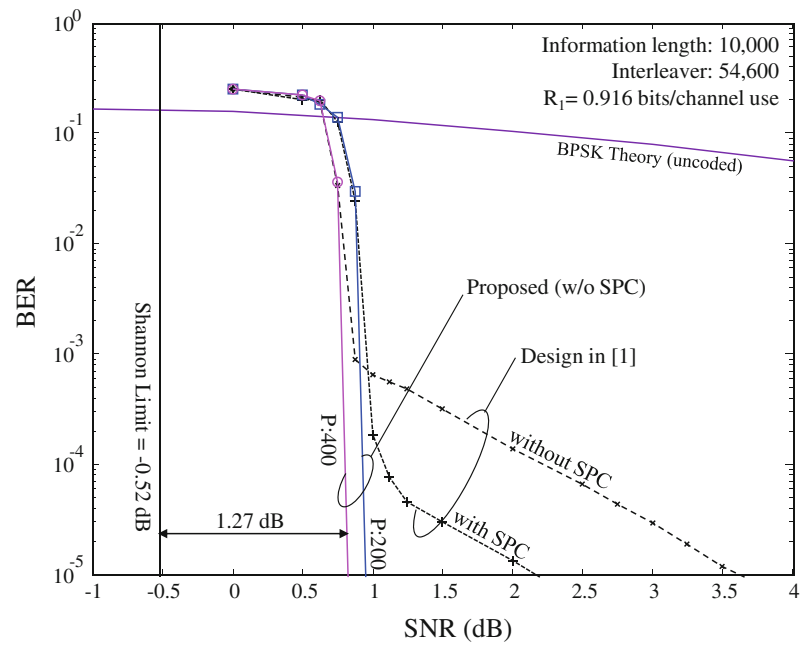

Fig. 7 BER performances for $\mathcal{R}_{1}=0.916$ bits/channel use of extended non-Gray 4-QAM

to $P=\{200,300,400,500\}$. In this paper we consider the degree distribution empirically obtained and used in $[1,4]$ for extended 4-QAM with $\ell=5$.

The performances are evaluated for two cases with the parameters chosen as in Table 1, which are the same as those used in the EXIT chart analysis presented in Sect. 3.

By eliminating the SPC codes and adding the rate-1 ACC with doping ratio of $(1: P)$, the proposed EM BICM-ID provides better BER performance and higher bits/channel use, which are:

Case 1: $\mathcal{R}_{1}=\frac{\ell}{\sum_{i=1}^{2} a_{i} \cdot d_{v_{i}}}=0.916\left(\mathcal{R}_{1, \text { Ref.[1] }}=0.904\right) \mathrm{bits} /$ channel use,

Case 2: $\mathcal{R}_{2}=\frac{\ell}{\sum_{i=1}^{3} a_{i} \cdot d_{v_{i}}}=1.299\left(\mathcal{R}_{2, \text { Ref.[1] }}=1.286\right) \mathrm{bits} / \mathrm{channel}$ use, both with $\ell=5$.

Figure 7 shows, for comparison, BER performances with Case 1 of the proposed and Ref. [1]'s techniques with maximum 50 iterations. The BER curve of EM BICM-ID with SPC exhibits minor improvement, however, the error floor still remains. On the contrary, the proposed technique with ACC and doping ratio of (1:200) or (1:400), can completely eliminate the error floor even without the use of SPC. Assuming the Gaussian codebook, the Shannon limit for $\mathcal{R}_{1}=0.916 \mathrm{bits} / \mathrm{channel}$ use is

$$
\begin{aligned}
\operatorname{SNR}_{\text {lim }}\left(\mathcal{R}_{1}\right) & =10 \log _{10}\left(2^{\mathcal{R}_{1}}-1\right), \\
& =-0.52 \mathrm{~dB} .
\end{aligned}
$$




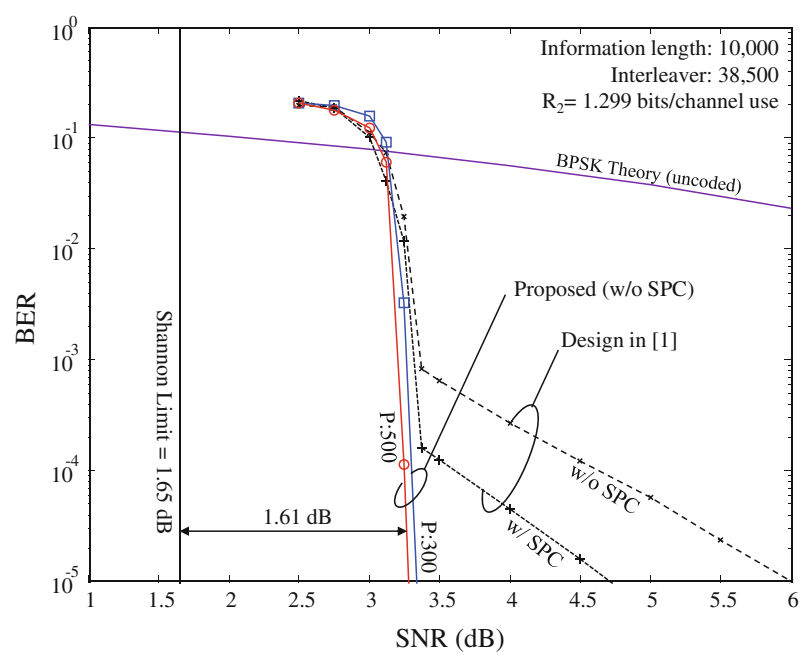

Fig. 8 BER performances for $\mathcal{R}_{2}=1.299$ bits/channel use of extended non-Gray 4-QAM

BER performance for the Case 2 is presented in Fig. 8 for code rate $\mathcal{R}_{2}$ and doping ratio of (1:300) and (1:500), where the Shannon limit for $\mathcal{R}_{2}=1.299$ bits/channel use is

$$
\begin{aligned}
\operatorname{SNR}_{\text {lim }}\left(\mathcal{R}_{2}\right) & =10 \log _{10}\left(2^{\mathcal{R}_{2}}-1\right), \\
& =1.65 \mathrm{~dB} .
\end{aligned}
$$

It can be observed from Figs. 7 and 8, that the proposed techniques improve not only the spectrum efficiency by removing the necessity of SPC but also the BER performance by eliminating the error floor while keeping the near-Shannon limit performance.

The performance improvements achieved by $P=400$ and $P=500$ for $\mathcal{R}_{1}$ and $\mathcal{R}_{2}$, respectively, with their degree allocations shown in Table 1 are the largest among other settings tested. It should be noted here that instead of using empirically obtained degree distribution, optimal node degree distribution $d_{v_{i}}$ can be determined by the linear programming (LP) technique, such as that presented in [5], to further improve the performance of the proposed EM BICM-ID system. This is left as further research.

\section{Conclusion}

This paper has proposed a very simple BICM-ID technique using a combined use of IRC and doped ACC assisted extended non-Gray mapping. The proposed techniques provide: (a) higher spectrum efficiency by removing the necessity of SPC, (b) better matching between the demapper and the decoder and hence eliminating the error floor caused by EXIT curves intersection before they reach the $(1,1)$ MI point. The numerical result for an exemplifying design confirms that the turbo-cliff is achievable within $1.27 \mathrm{~dB}$ away from the Shannon limit for 0.916 bits/channel use with 3-bit $(\ell=5)$ extended 4-QAM.

Acknowledgment We would like to acknowledge Dan Zhao for developing the simulation software of the demapper part. 
Open Access This article is distributed under the terms of the Creative Commons Attribution Noncommercial License which permits any noncommercial use, distribution, and reproduction in any medium, provided the original author(s) and source are credited.

\section{References}

1. Zhao, D., Dauch, A., \& Matsumoto, T. (2009). BICM-ID using extended mapping and repetition code with irregular node degree allocation. In IEEE VTC-Spring (pp. 1-5). Barcelona.

2. Li, X., \& Ritcey, J. (1997). Bit-interleaved coded modulation with iterative decoding. IEEE Соттиnications Letter, 1(6), 169-171.

3. Henkel, P. (2006). Extended mapping for bit-interleaved coded modulation. In IEEE personal, indoor and mobile radio communications (PIMRC) (pp. 1-4). Helsinki.

4. Zhao, D., Dauch, A., \& Matsumoto, T. (2009) Modulation doping for repetition coded BICM-ID with irregular degree allocation. In ITG wireless smart antenna. Berlin.

5. Fukawa, K., Zhao, D., Dauch, A., Tolli, A., \& Matsumoto, T. (2010). Irregular repetition and single parity check coded BICM-ID using extended mapping: Optimal node degree allocation. In ICST conference on communications and networking in China (CHINACOM), Beijing. (Invited).

6. Pfletschinger, S., \& Sanzi, F. (2006). Error floor removal for bit-interleaved coded modulation with iterative detection. IEEE Transactions on Wireless Communications, 5(11), 3174-3181.

7. Durisi, G., Dinoi, L., \& Benedetto, S. (2006). eIRA codes for coded modulation systems. In IEEE ICC (pp. 1125-1130). Istanbul.

8. ten Brink, S. (2001). Convergence behavior of iteratively decoded parallel concatenated codes. IEEE Transactions on Communications, 49, 1727-1737.

9. Berrou, C., Glavieux, A., \& Thitimajshima, P. (1993). Near Shannon limit error-correction coding and decoding: Turbo code. In IEEE International conference on communication (ICC) (pp. 1064-1070). Geneva.

\section{Author Biographies}

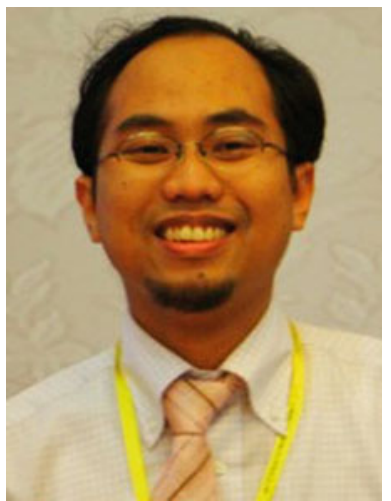

Khoirul Anwar graduated (cum laude) from the department of Electrical Engineering (Telecommunications), Institut Teknologi Bandung (ITB), Bandung, Indonesia in 2000. He received Master and Doctor Degrees from Graduate School of Information Science, Nara Institute of Science and Technology (NAIST) in 2005 and 2008, respectively. Since then, he has been appointed as an assistant professor in NAIST. He received best student paper award from the IEEE Radio and Wireless Symposium 2006, California-USA, and Best Paper of Indonesian Student Association (ISA 2007), Kyoto, Japan in 2007. Since September 2008, he is with the School of Information Science, Japan Advanced Institute of Science and Technology (JAIST) as an assistant professor. His research interests are network information theory, error control coding, iterative decoding and signal processing for wireless communications. 


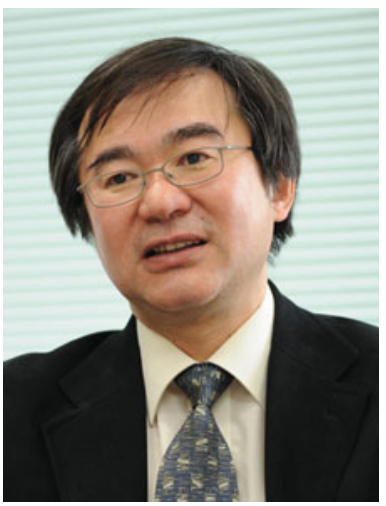

Tad Matsumoto received his B.S., M.S., and Ph.D. degrees from Keio University, Yokohama, Japan, in 1978, 1980, and 1991, respectively, all in electrical engineering. He joined Nippon Telegraph and Telephone Corporation (NTT) in April 1980. Since he engaged in NTT, he was involved in a lot of research and development projects, all for mobile wireless communications systems. In July 1992, he transferred to NTT DoCoMo, where he researched Code-Division MultipleAccess techniques for Mobile Communication Systems. In April 1994, he transferred to NTT America, where he served as a Senior Technical Advisor of a joint project between NTT and NEXTEL Communications. In March 1996, he returned to NTT DoCoMo, where he served as a Head of the Radio Signal Processing Laboratory until August of 2001; He worked on adaptive signal processing, multipleinput multiple-output turbo signal detection, interference cancellation, and space-time coding techniques for broadband mobile communications. In March 2002, he moved to University of Oulu, Finland, where he served as a Professor at Centre for Wireless Communications. In 2006, he served as a Visiting Professor at Ilmenau University of Technology, Ilmenau, Germany, funded by the German MERCATOR Visiting Professorship Program. Since April 2007, he has been serving as a Professor at Japan Advanced Institute of Science and Technology (JAIST), Japan, while also keeping the position at University of Oulu. Prof. Matsumoto has been appointed as a Finland Distinguished Professor for a period from January 2008 to December 2012, funded by the Finnish National Technology Agency (Tekes) and Finnish Academy, under which he preserves the rights to participate in and apply to European and Finnish national projects. Prof. Matsumoto is a recipient of IEEE VTS Outstanding Service Award (2001), Nokia Foundation Visiting Fellow Scholarship Award (2002), IEEE Japan Council Award for Distinguished Service to the Society (2006), IEEE Vehicular Technology Society James R. Evans Avant Garde Award (2006), and Thuringen State Research Award for Advanced Applied Science (2006), 2007 Best Paper Award of Institute of Electrical, Communication, and Information Engineers of Japan (2008), Telecom System Technology Award by the Telecommunications Advancement Foundation (2009), and IEEE Communication Letters Exemplifying Reviewer Award (2011). He is a Fellow of IEEE and a Member of IEICE. He is serving as an IEEE Vehicular Technology Distinguished Lecturer during the term July 2011-June 2013. 